\title{
Measurement of Field Distortion in Free-Air Ionization Chambers by Analog Method ${ }^{1}$
}

\author{
William Miller ${ }^{2}$ and Robert J. Kennedy ${ }^{3}$
}

\begin{abstract}
A two dimensional analog of a free-air ionization chamber for the measurement of field distortion was obtained by painting conducting lines on resistance paper to represent cross sections of the electrodes. Guard wires were simulated by driving record player needles into the paper. The volume of the chamber from which charge was collected was related to the resistance between two of the lines on the paper, and small changes in this volume could be determined accurately by observing resistance changes. Grounded plates external to the chamber were simulated by additional lines painted on the paper, and the effect of these plates on the collecting volume was determined by measuring a change in resistance as the potential of these (simulated) external plates was changed. Measurements were also made for guards in the form of double wires and strips. There is some evidence from measurements on three dimensional ionization chambers that the top and bottom of the surrounding grounded X-ray shield tends to decrease the effects of distortion caused by the ends of the X-ray shield, so that the data obtained here would represent an upper limit to the error for the indicated chamber cross section.
\end{abstract}

\section{Introduction}

Of fundamental interest in connection with X-ray measurements is the dose, a quantity that is closely correlated with biological damage. For X-rays with peak energies below $3 \mathrm{Mev}$, the roentgen is the unit of dose, and is defined in terms of the ionization produced by the X-rays in a given mass of air [1]. ${ }^{4}$ A direct measurement of the roentgen is achieved with the aid of a free-air ionization chamber, wbich is designed to collect the ionization produced in a defined region of air by a collimated beam of X-rays. One of the problems associated with the use of a free-air chamber is the accurate delineation of this "collection volume."

The electrode system in a free-air chamber is often a guarded parallel plate condenser with the vertical planes of the electrodes parallel to the direction of the X-ray beam. The collimated X-ray beam passes horizontally through the center of the condenser. One plate of the condenser is positioned to one side of the beam and maintained at a high potential in order to sweep out the ionization produced in air by the beam. The plate on the opposite side is divided into three regions. The ionization collected by the central region (collecting plate) is measured. The other two are guard plates to reduce the field distortion. The small gap between the collector and each of the guard plates extends to the full height of the plates.

Some of the geometrical parameters of the chamber must meet requirements imposed by physical propties of the radiation. For example, the separation of the plates will depend on the range of the electrons which are ejected by the incident beam of X-rays. The range of the electrons will also place a minimum requirement on the beight of the plates. Another restriction on the chamber is that the distance from

This work was partially supported by the United States Atomic Energy Commission.

${ }^{2}$ Now with Naval Research Laboratory, Washington, D. C

3 Now with Food and Drug Administration, Dept. of Health, Education, and Welfare, Washington, D. C

${ }_{4}^{4}$ Figures in brackets indicate the literature references at the end of this paper the collimater to the front of the collecting region be sufficient for electronic equilibrium to be established. Physical requirements such as these have been studied in detail in connection with the $250 \mathrm{kv}$ and $500 \mathrm{kv}$ standard free-air chambers at the Bureau by Attix and DeLaVergne [2] and by Wyckoff and Kirn [3].

Another set of limitations on the chamber design is related to the problem of electrostatic field distortion. If the chamber is to be used as an absolute measuring device one must have accurate information about the volume from which the ionization is collected. The diaphragm of the chamber defines the area of the beam but the longitudinal dimension of this volume is determined by the electrostatic force lines reaching the vertical edges of the collector plate. If the electrostatic field is sufficiently wellguarded, the field will be uniform, and the length of the collecting volume known precisely. However, it is usually necessary to restrict the size of the ionization chamber, and to surround it with a lead shield at ground potential to minimize the background from stray X-rays, and to act as an electrostatic shield. The distortion produced by this shield can effectively decrease the dimension of the collecting volume in the direction of the X-ray beam, and cause a corresponding change in the ionization current collected. It is this type of field distortion which is of prime interest here, and the bulk of the work to be described was designed to determine the magnitude of this distortion for various chamber configurations.

A lead shield surrounding the ionization chamber can also change the effective dimension of the collecting volume in a vertical direction. This distortion is less important because the ionization density falls off rapidly as one moves radially off the beam. To determine its effect the distortion data presented below must be combined with data on the radial distribution of the ionization [2]. However, there is some evidence, discussed below, that indicates that the perpendicular distortion may affect the length of the collecting region. 
A type of field distortion which is not due to the shield around the chamber, is the bowing out of the electrostatic lines of force which occurs at the edges of the plates in a condenser. It will be shown that this effect is very small for the well-guarded chambers considered here.

Examples of early investigations of field distortion are those of Duane and Lorenz [4] and Failla [5]. Duane and Lorenz isolated the effect of an external grounded plate by observing the change in ionization current as this plate was successively connected to the ground electrode of the chamber and the high voltage electrode. The measurement was carried out for a particular chamber configuration, and hence did not yield sufficient data to determine design requirements.

Failla [5] has investigated the field distortion in a free air chamber designed for use with X-rays generated at about $100 \mathrm{kv}$. The plates were $42 \mathrm{~cm}$ long and $22 \mathrm{~cm}$ high, and the collector was divided into 11 strips $22 \mathrm{~cm}$ high and about $3.9 \mathrm{~cm}$ wide. The beam entered the chamber horizontally. The beam diameter was small compared to $22 \mathrm{~cm}$, and since the photoelectrons and Compton electrons did not produce significant ionization at distances of the order of $11 \mathrm{~cm}$, the field distortion at the top and bottom of the chamber for small plate separators did not affect the ionization current collected. The chamber was surrounded by a grounded shield, (except for holes to let the beam through) and the dimension of this shield along the beam direction was $53 \mathrm{~cm}$. When the plate separation was sufficiently small, the ionization currents to several of the collector strips near the center were equal, and this was interpreted as indicating that the field was uniform in this region. ${ }^{5}$ When the plate separation was increased the number of plates for which the currents were equal decreased. In this way Failla estimated what fraction of the region between the electrode system was in a uniform field, as a function of the ratio of electrode length to plate separation. He estimated, for example, that if the plate separation was one-tenth of the total length of the electrodes the field was uniform for 80 percent of the region between the plates. This uniform region was reduced to about 35 percent when the separation was increased to one-fifth of the electrode length and when the separation reached one-half of the plate length (i. e., at a separation of $21 \mathrm{~cm}$ ) the field was not even uniform over a single collector plate. Although this study was carried out with the ends of the grounded box at a given distance $(5.5 \mathrm{~cm})$ from the edges of the electrodes, it is evident that the field distortion increases as this distance is decreased.

Free-air chambers are currently built for measurements on X-rays with peak energies well above the $100 \mathrm{kv}$ used by Duane and Lorenz and by Failla.

The fact that several strips collected the same ionization would not in itself prove that there is no distortion, since the distortion error might be the same in each of these strips. Failla's interpretation was supported by the fact that the current to the outer strip remained constant as the plate separation was increased. However, Attix has recently raised the point that Failla's plate separation was inadequate for the electron range involved. This would cause an increase in ionization current with plate separation, which would tend to cancel the losses the equality of currents to several collector strips assures that there is no field distortion
The plate separation must be greater for these higher peak energies, and this makes the distortion problem more serious. For example, one might consider a $500 \mathrm{kv}$ chamber to be built as a scaled up version of the chamber studied by Failla [5]. The required separation of the electrodes is such that each guard plate would have to be well over a meter long, if the field is to be uniform over a sufficient region for collection. In order to achieve sufficient field uniformity with reasonable electrode lengths, free-air chambers are usually provided with a set of guard wires between the edges of the parallel condenser plates. Each wire is in a plane parallel to the condenser plates. The wires are uniformly spaced and their potential is uniformly graded by a resistance network. Studies of the effect of guard wires on field uniformity were made by Failla [5] and by Taylor and Singer [6]. This guarding has been carried further in the standard free-air chambers at the Bureau. The $250-\mathrm{kv}$ chamber is provided with double guard wires and the $500-\mathrm{kv}$ standard has a combination of guard wires and strips.

To determine what the field distortion is in a given chamber, one could carry out measurements, similar to those of Duane and Lorenz and Failla, on the actual chamber. Alternatively one might construct scale models and determine their capacitance. The deviation of the measured capacitance from the computed undistorted capacitance could be used as a measure of the field distortion. However, free-air chambers do not usually have guard plates above and below the collector. This is not considered to be necessary, as the ion density in this region is very low, so a relatively large distortion is acceptable there. Capacity deviation would be due principally to distortion in this region. Moreover if scale models are made, the distortion determined by ionization methods would be more directly applicable. Thus, neither of these methods seems practical when the effect of a number of variables is desired. To permit distortion measurements to be made for a wide variety of configurations, an analog device was set up. Since the standard free-air chambers at NBS are being constructed to have an over-all accuracy of 0.5 percent, the analog device for measuring the effect of field distortion was designed to have an accuracy of 0.1 percent.

It should be noted that the measurements described here are two-dimensional, so that the results are strictly applicable only when the plate heights are effectively infinite. In an actual chamber, the plates will be of finite height, and there will be grounded plates above and below the chamber. Guard wires running along the top and bottom of the chamber decrease the distortion produced by the top and bottom of the surrounding X-ray shield. It was pointed out earlier that one of the effects of ground above and below the chamber is a vertical deflection of the lines of force, and that this tends to be unimportant because the ionization falls off rapidly as one moves radially out from the beam center. However, it is also possible that the top and bottom of the X-ray shield will affect the electrostatic field at the ends of the cham- 
ber. Evidence of such an effect was obtained with the $250-\mathrm{kv}$ chamber and will be described below.

\section{Method of Measurement}

The effect of a grounded plate on the electrostatic field inside the chamber has been schematized in figure 1 , which is a cross section of the chamber in a plane perpendicular to the electrodes, and contains the X-ray beam. The collector is represented by $\mathrm{C}$, the guard plates by $\mathrm{G}$, the high voltage plate by $\mathrm{H}$, the guard wires by W, and grounded shield by $\mathrm{B}$. The presence of the box, B, causes the lines of force leaving the edge of the collector plate to flare in, as indicated by the dashed lines $\mathrm{F}^{\prime}$. (The lines $\mathrm{F}^{\prime}$ and $\mathrm{F}^{\prime \prime}$ are purely schematic, and are designed to show the direction of shift of the lines of force rather than a detailed shape.) For the configurations to be studied here, these lines of force would be normal to the electrodes (as shown by the dashed lines F) if the grounded box were removed. ${ }^{6}$

To determine the decrease in collecting volume caused by the presence of B, (i. e., by the shift of the lines from $\mathrm{F}$ to $\mathrm{F}^{\prime}$ ) one could simulate the cross section of the chamber in an electrolytic tank [7], and plot the lines of force. However, it has proven difficult to achieve an accuracy of 1 percent in the field plots obtained with an electrolytic tank [7], so that this technique is of limited use here.

The desired accuracy was achieved by making a more direct measurement of the collecting volume. If the region between the collector and guard plate system and the high voltage plate were filled with a medium of uniform resistivity, the resistance between the collector plate and the high voltage plate would be related to the collecting volume. This may be seen by reference to figure 1, where the length of the collector plate has been specified as $L ; x$ is the distance from a point in the chamber to the plane of the collector plate, and $\delta(x)$ represents the "displacement" of the line of force $\mathrm{F}^{\prime}$ from $\mathrm{F}$, measured parallel to the electrodes. $(\delta(x)$ will be considered positive if the lines $\mathrm{F}^{\prime}$ are further apart than $L$ at that value of $x$.) If one considers a thickness $\mathrm{T}$ of the resistive medium, and its resistivity is

${ }_{6}$ The question of whether or not the field is uniform when the grounded shield is not present is discussed further in the next section.

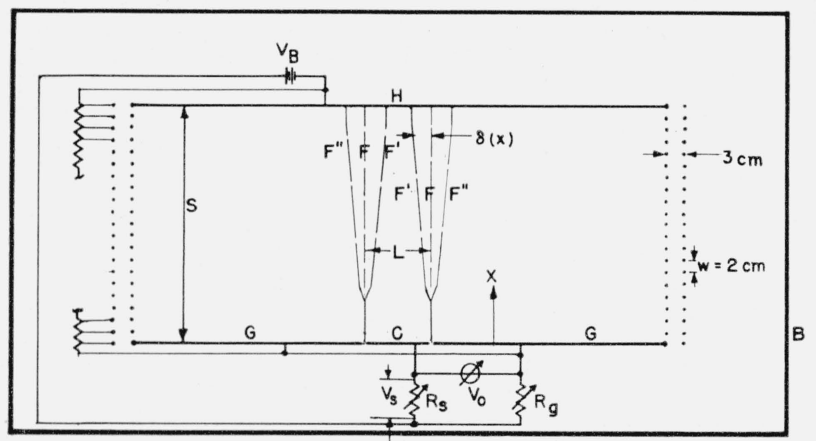

Figure 1. Schematic cross-section of free air ionization chamber. $\rho$, then the resistance between the collector and high voltage plates is

$$
R=\int_{0}^{S} \frac{\rho d x}{T[L+2 \delta(x)]}
$$

where $S$ is the separation between the plates. If $\delta<L$, the denominator can be expanded:

$$
\frac{1}{L+2 \delta(x)} \cong \frac{1}{L}\left(1-\frac{2 \delta(x)}{L}+\frac{[2 \delta(x)]^{2}}{L^{2}} \cdots\right) \text {. }
$$

For $\delta / L$ of the order of a few percent or less i. e., if the distortion is small, only the first two terms need be retained, and (1) becomes

$$
R \cong \frac{\rho}{T L}\left[S-\int_{0}^{S} \frac{2 \delta(x) d x}{L}\right]
$$

If $R_{0}$ is defined as the resistance between $C$ and $H$ when the electrostatic field is uniform, then $R_{0}=\frac{\rho S}{T L}$ and eq (3) becomes:

$$
R=R_{0}-R_{0} \frac{\Delta V}{V_{0}}
$$

Where $V_{0}=L S T$, and represents the collecting volume when the field is uniform, and $\Delta V$ represents the change in this volume when the lines $\mathrm{F}$ shift to $\mathrm{F}^{\prime}$. Equation (4) may be written

$$
\frac{\Delta R}{R_{0}}=-\frac{\Delta V}{V_{0}}
$$

The resistance measurements could be made by inserting plates and wires into an electrolytic tank, to simulate a cross section of the chamber, and measuring resistances between the collector and high voltage plates. Since it would have required an elaborate mechanical setup to permit variation of the chamber parameters while maintaining accurate alignment of the components in the electrolytic tank, this method did not show promise.

A suitable resistive medium was found in the form of a conducting paper used for electrical recording. [8] Cross sections of the electrodes were represented by lines of conducting paint, which were applied to the paper in the form of silver in a butyl acetate carrier. In all cases the separation, S, was set at $40 \mathrm{~cm}$ (see fig. 1). The guard wires were simulated by driving record player needles through the paper. The cross section of the needles in the plane of the paper was a circle with a diameter of $0.5 \mathrm{~mm}$. The spacing between the needles was $2 \mathrm{~cm}$, so that there were 21 needles at each end of the chamber, except in the double guard wire case, when an additional line of needles, $3 \mathrm{~cm}$ outside the first line, was provided at each end of the chamber. Guard strips were also simulated by painting a fine conducting line between each pair of needles in the double wire setup. A 6-volt storage battery supplied a potential to the condenser. 
The same potential, together with a dividing network, uniformly graded the potential to the needles. The variable resistors $R_{s}$ and $R_{g}$ permitted independent measurement of the current to the collector, while maintaining the collector and guard plates at the same potential.

In principle, the quantity $\Delta R / R_{0}$, in eq (5), could be determined by first measuring the resistance between $\mathrm{C}$ and $\mathrm{H}$, with no outside shield, $\mathrm{B}$, drawn on the paper, and then repeating the resistance measurement with $\mathrm{B}$ painted in and connected to ground. This method would be rather time consuming, and subject to drift errors. ${ }^{7}$ The measurement of $R$ and $R^{\circ}$ can be made in a few seconds, if one takes advantage of symmetry. If the presence of the grounded box causes the lines of force to shift from $\mathrm{F}^{\mathrm{T}}$ to $\mathrm{F}^{\prime}$, in figure 1 , then the presence of a box, B, at the same potential as $\mathrm{H}$ would cause the lines $\mathrm{F}$ to shift to some position such as $\mathrm{F}^{\prime \prime}$. The decrease in collecting volume caused by the shift from $\mathrm{F}$ to $\mathrm{F}^{\prime}$, will be almost exactly equal to the increase resulting from the shift from $\mathrm{F}$ to $\mathrm{F}^{\prime \prime}$. Thus, the change in resistance between $\mathrm{C}$ and $\mathrm{H}$, which is observed when the potential on B is changed from ground to the same potential as $\mathrm{H}$, is equal to $2 \Delta R$, and the average of the two values measured is $R_{0}$. This procedure is analogous to the one used by Duane and Lorenz [4], with an actual chamber, but there is more flexibility in using resistance paper, since the chamber cross section can be altered by merely painting a line on the paper.

It was found, during the course of measurement, that the distortion produced in a given chamber ground-plate configuration is quite sensitive to the diameter of the guard wires. A study has been made to determine the variation of distortion with wire diameter, and is summarized below.

Although all the data presented below was obtained with a (simulated) plate separation of $40 \mathrm{~cm}$, it is evident that all the dimensions may be scaled so as to provide results which apply to other plate separations.

\section{Results}

Figures $2 \mathrm{a}, 2 \mathrm{~b}$, and $2 \mathrm{c}$ summarize the distortion data for a single line of guard wires at each end of the chamber. Each curve pertains to a given distance between the ends of the ground box and the edges of the guard plates, and the four curves in each figure apply to a given size collector. Figures $3 \mathrm{a}, 3 \mathrm{~b}$, and $3 \mathrm{c}$, are the corresponding data for two sets of guard wires at each end of the chamber. Each of the guard wires in the extra set is presumed to be at the same potential as the corresponding wire in the original set of guard wires, but placed $3 \mathrm{~cm}$ further out.

Figure 4 compares the distortions obtained in chambers guarded by single wires, double wires, and strips $3 \mathrm{~cm}$ wide. In each case, the guard

The resistance of four samples of Teledeltos paper tested showed a temperature coefficient $-0.1 \% /{ }^{\circ} \mathrm{C}$ (at $0 \% \mathrm{R} . \mathrm{H}$. and $20^{\circ} \mathrm{C}$ ), and a relative humidity coefficient of $+1 \%$ per one percent increase in relative humidity (at $30^{\circ} \mathrm{C}$ and $50 \% \mathrm{R}$. $\mathrm{H}$.)
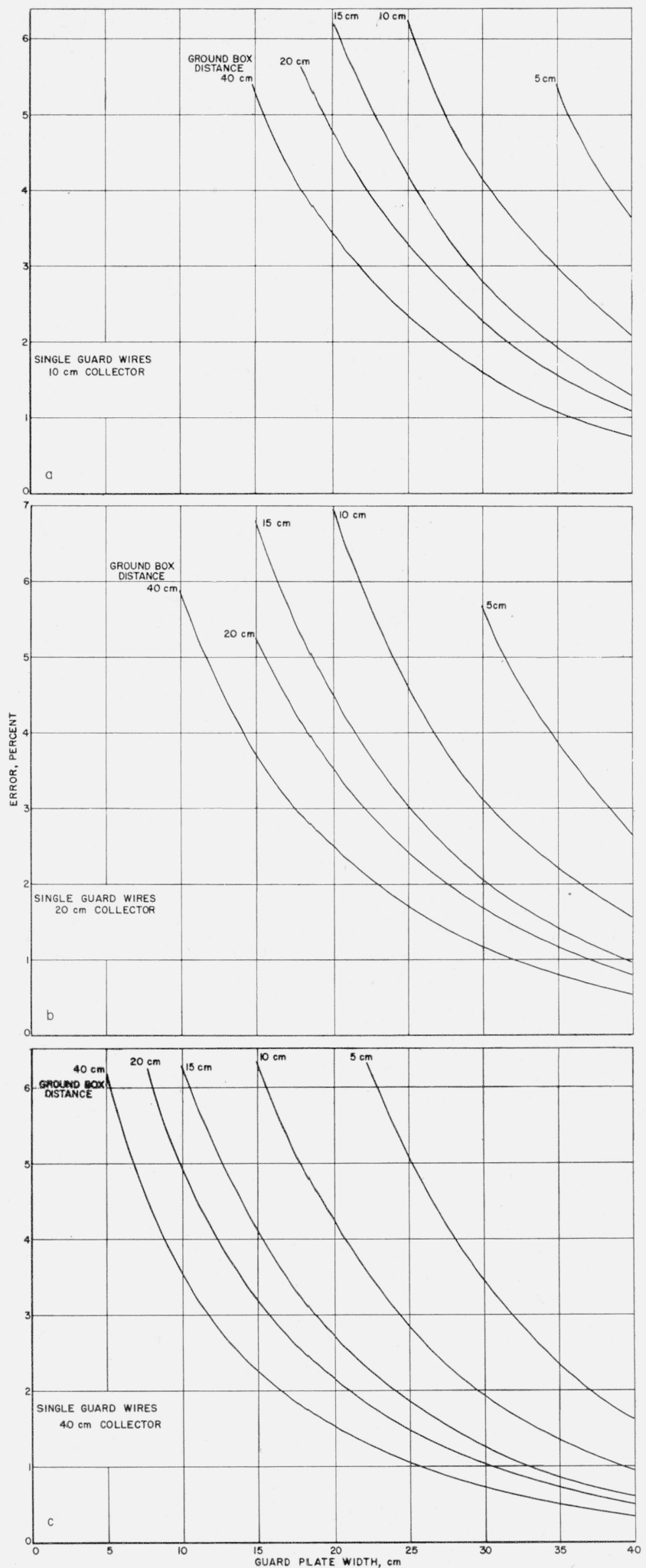

Figure 2. Error $\left(\frac{=100 \Delta R}{R_{\mathrm{o}}}\right)$ in collecting volume induced by the presence of the grounded box.

Plate separation is $40 \mathrm{~cm}$; collector length is $10 \mathrm{~cm}$ in (a), $20 \mathrm{~cm}$ in (b), and 40 $\mathrm{cm}$ in (c). Each curve corresponds to given distance between end of guard plate and end of grounded box. The guard wires are $0.5 \mathrm{~mm}$ in diameter, arranged at intervals of $2 \mathrm{~cm}$ in single line at each end of chamber. 

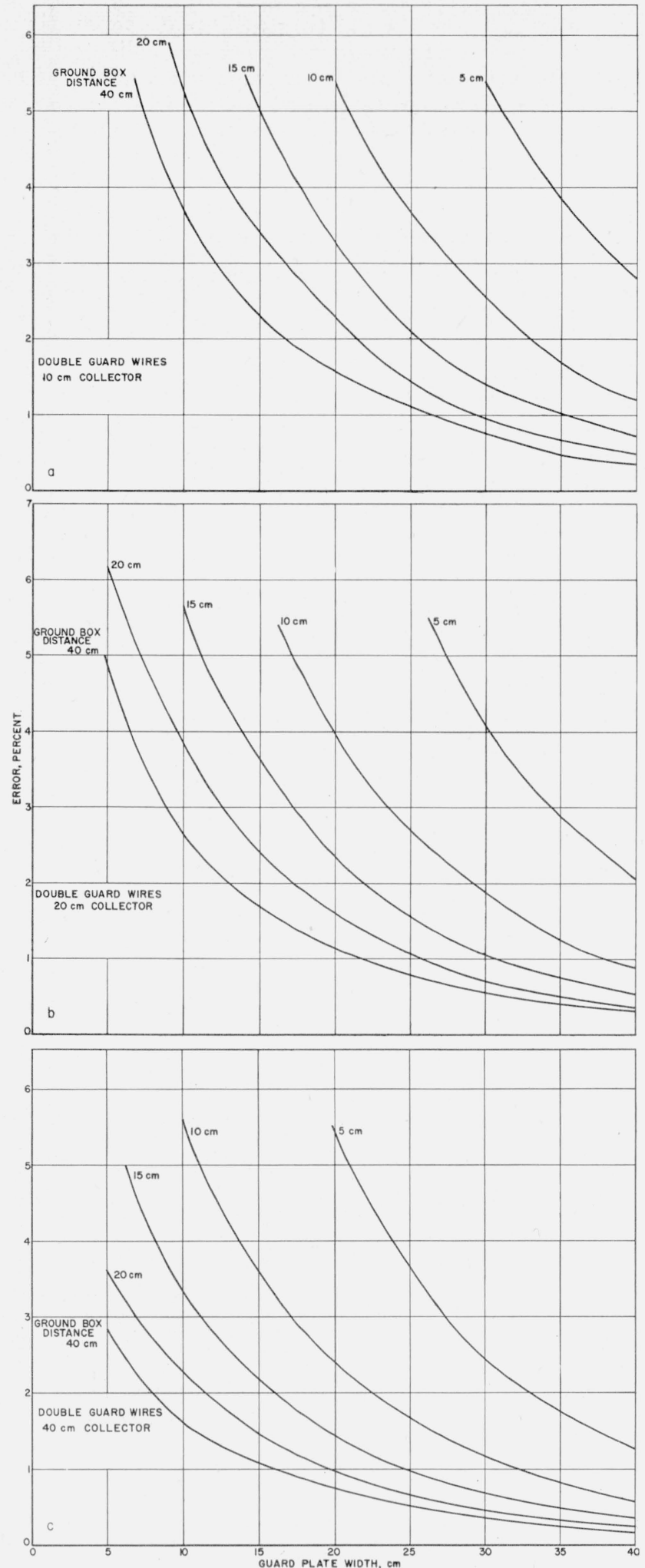

Figure 3. Error in collecting volume induced by the presence of the grounded box.

Plate separation is $40 \mathrm{~cm}$; collector length is $10 \mathrm{~cm}$ in (a), $20 \mathrm{~cm}$ in (b) and 40 $\mathrm{cm}$ in (c). Each curve corresponds to given distance between end of guard plate and end of grounded box. The guard wires are $0.5 \mathrm{~mm}$ in diameter, arranged in two lines, $3 \mathrm{~cm}$ apart, at each end of the chamber. The wires within each line are spaced at intervals of $2 \mathrm{~cm}$.

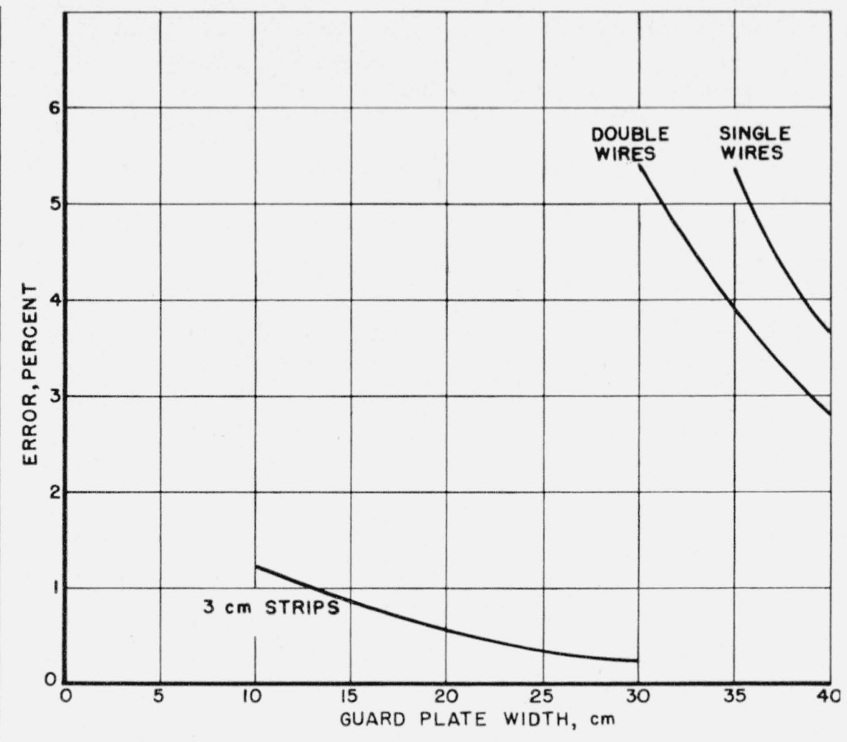

Figure 4. Comparisons of errors induced in collecting volume (by grounded box) when the chamber is guarded by single wires, double wires, and strips.

The plate separation was $40 \mathrm{~cm}$, and the collector plate was $10 \mathrm{~cm}$ long.

elements were spaced by $2 \mathrm{~cm}$, so that 21 sets were required at each end of the chamber. The measurement was carried out on the resistance paper by first inserting a single set of needles into the paper at each end of the chamber and measuring the the resistance between $\mathrm{C}$ and $\mathrm{H}$ with $\mathrm{B}$ at the high potential and at ground. A second set of needles was then inserted at each end, and each needle connected by a wire to its counterpart in the first set and the resistance between $\mathrm{C}$ and $\mathrm{H}$ again measured with B at the appropriate potentials. Lastly, each needle in the first set of guard wires was connected to the corresponding needle in the second set by a thin line of conducting paint on the paper (instead of the copper wire, which didn't touch the paper). The results indicate that the strips are much more effective in reducing distortion than are double or single wires.

It should be noted that the dimensions of the cross section of these strips on the paper were not accurately controlled because of flow of the silver paint on the paper. The small dimension of the cross section varied between 0.5 and $1 \mathrm{~mm}$, and makes these data uncertain by about a factor of two, as will be seen in the next paragraph. Even after allowance is made for this possible error, the improvement resulting from the use of strips is still evident. However, one drawback to the use of strips is that they can cause considerable absorption and scattering if they are in the X-ray beam. This difficulty was overcome in the $500 \mathrm{kv}$ chamber by using strips everywhere but in the X-ray beam, where the strips were replaced by double wires.

Figure 5 contains the results of a study of the effect of wire diameter on the effectiveness of the guard wire system. The diameter of the cross section of the needle was effectively increased by applying a small amount of conducting paint to the shaft of the needle, and letting it spread on the 


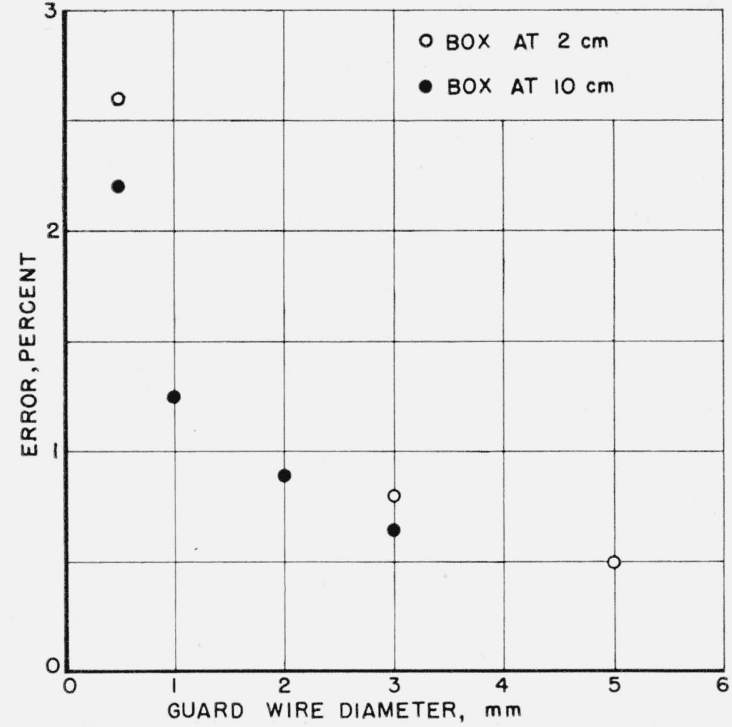

FIGURE 5. Effect of guard wire diameter on error induced in collecting volume by grounded box.

Plate separation was $40 \mathrm{~cm}$, collector plate was $10 \mathrm{~cm}$ long, guard plate was $40 \mathrm{~cm}$ long and with a single line of guard wires at each end of chamber at intervals of $2 \mathrm{~cm}$.

resistance paper. The sizes of the spots could not be precisely controlled, and had a \pm 20 percent fluctuation, so that the results are relatively qualitative. However, it can be seen that the wire diameter is a critical factor in determining field distortion.

The data presented thus far were obtained by observing a change in resistance as the potential on the external ground was changed from that of the ground place to that of the high voltage plate. These results indicate only the effect of the external ground on the field. It is still necessary to determine whether there is any significant field distortion due to causes other than the presence of the grounded shield.

When the external X-ray shield is placed first at ground potential and then at the potential of the high voltage plate, the lines of force move from a position such as $\mathrm{F}^{\prime}$ to one such as $\mathrm{F}^{\prime \prime}$ (fig. 1). These lines of force straddle the position, $\mathrm{F}$, that they would take if the box, B, were not present. It has been pointed out, that for the small distortions which have been considered, the area between $\mathrm{F}^{\prime}$ and $\mathrm{F}$ is equal to the area between $\mathrm{F}$ and $\mathrm{F}^{\prime \prime}$, so that the average value of the resistances obtained when the box is at ground and at high potential represents a collecting volume contained between the lines $\mathrm{F}$. The question to be considered here is whether the lines $\mathrm{F}$ correspond to a uniform field. One straightforward way to determine this is to measure the resistance when the field is constrained to be uniform, and compare it with the average of the resistance obtained on reversing potential on the shield.

To obtain a uniform field in the region between collector and high voltage plate, a separate chamber was drawn on resistance paper with guard plates whose length was several times the plate separation. The resistance was measured between collector and high voltage plate, and then measured again after the guard plates were lengthened. The resistance remained the same after the guards were lengthened. This then provided a method of determining the resistance corresponding to a uniform field, and for setting up a standard with which the average re sistance determined by potential reversal could be compared. This comparison was carried out in several cases, and there was no detectable difference between the uniform field case and the average between the $\mathrm{F}^{\prime}$ and $\mathrm{F}^{\prime \prime}$ configuration shown in figure 1. It appears safe to conclude that in the chamber configurations studied here the only significant cause of field distortion is the grounded box.

One might also question whether or not the wires themselves produced a distortion of the field in the collection region. If so, the average of the resistance obtained with the box at ground potential and at the potential of $\mathrm{H}$ (fig. 1) should vary with the num ber of guard wires in a given geometry. Distortions were measured for different guard wire spacings, W, on each of several sheets. For small distortions (within the approximation of eq. 3) changing the number of guard wires had no effect on the average resistance. Therefore, the wires themselves do not produce a distortion in the collector region if the overall distortion is small.

A comparison of the "two-dimensional" data obtained with the resistance paper with a distortion measurement made in the 250 -kv chamber, indicates the extent of the three dimensional effect mentioned earlier. The chamber had a plate separation of 20 $\mathrm{cm}$, a $10-\mathrm{cm}$ collector, two $18-\mathrm{cm}$ guard plates, a single set of guard wires of 0.5 diameter, spaced 1 $\mathrm{cm}$ apart, and a grounded box with ends approximately $4 \mathrm{~cm}$ from the guard wires. The top and bottom of the grounded box were about $2 \mathrm{~cm}$ from the top and bottom of the plate system. The resistance paper measurements would indicate a field distortion error of about 1 percent for this configuration if the appropriate adjustment is made to account for the wire diameter. The distortion in the chamber was measured by observing the change in ionization current as the potential on the box was reversed. When the collecting region was shielded from the top and bottom of the box with a single set of guard wires, the field distortion error was 0.3 percent. When the collecting region was shielded from the top and bottom of the box with a double set of guard wires (thereby reducing the effect of top and bottom of the box and making the chamber more nearly "two dimensional"), the field distortion error was 0.7 percent. A similar trend was observed in the $250-\mathrm{kv}$ chamber when the ends of the box were guarded with double wires, and the distortion measured with the top and bottom guarded first by double wires and then by strips. It is clear, then, that the field distortion in an idealized two-dimensional configuration, as measured by the resistance paper, provides an overestimate of the distortion in a threedimensional geometry. 


\section{Conclusion}

Adequate data are presented for determination of field distortion error in free-air ionization chambers of usual geometry. For a given set of chamber dimensions, the field distortion is found to be strongly dependent on the size of the guarding elements at the end of the chamber. With guard plate lengths approximately equal to the plate separation, and a grounded shield around the chamber only slightly larger than the plate system, the field distortion can be kept well below 0.5 percent, by use of suitable guard elements.

The resistance measuring techniques described will detect volume changes of less than 0.1 percent. It should be remembered, however, that because the measurements were made on a two-dimensional analog of an ionization chamber, they provide overestimates of the errors incurred in an actual three-dimensional chamber.

\section{References}

[1] Nucleonics 12, No. 1, 11 (1954).

[2] F. H. Attix and L. DeLaVergne, Radiology 63, 853 (1954).

[3] H. O. Wyckoff and F. S. Kirn, J. Research NBS (in preparation).

[4] W. Duane and E. Lorenz, Am. J. Roentgenol. 19, 461 (1928).

[5] G. Failla, Am. J. Roentgenol. 21, 47 (1929).

[6] L. S. Taylor and G. Singer, BS J. Research 5, 507 (1930) RP211.

[7] G. Liebman, Advances in Electronics 2, (1950).

[8] Western Union Technical Review 2, No. 4, (1948).

Washington, July 5, 1955. 